\title{
LA INVESTIGACIÓN SOCIAL UNIVERSITARIA: REDES DE CONOCIMIENTO EN CHIAPAS
}

\author{
Teresa Pacheco Méndez
}

kat_tpm@yahoo.es

Instituto de Investigaciones Sobre la Universidad y la Educación

Universidad Nacional Autónoma de México

\section{RESUMEN}

Entre muchas de las prácticas incorporadas y reconocidas en el oficio de la investigación ${ }^{1}$ en ciencias sociales figura la citación. Se trata de un recurso que incluso ha llegado a ser considerado como requisito indispensable de todo trabajo académico que se precie de calidad y de rigor científico. No obstante, las formas bajo las cuales ha evolucionado esta práctica han demostrado que lejos de garantizar resultados innovadores, la citación pone en evidencia la capacidad de una comunidad académica para vincularse local y globalmente gracias a las redes de comunicación y de conocimiento que se conforman a través de dicha práctica.

Palabras clave: investigación social, redes de conocimiento, Chiapas.

\begin{abstract}
Citation is used in many of the practices incorporated and recognized in social sciences research. It is a resource that is even considered to be an indispensable requirement in all academic work characterized by scientific rigor and quality. However, the ways in which this practice has evolved have demonstrated that, far from guaranteeing innovative results, citation reveals the capacity of an academic community to create links locally and globally as a result of the communication and knowledge networks established through this practice.
\end{abstract}

Key words: social research, knowledge networks, Chiapas. 


\section{INTRODUCCIÓN}

Entre los principales obstáculos a los que se enfrentan las comunidades académicas afines a las ciencias sociales para posicionarse y ajustar el alcance de sus redes de conocimiento entre lo local y lo global, figura la falta de acuerdos con respecto a lo que puede considerarse un conocimiento verdaderamente innovador de otro que no lo es. Las diferencias se establecen por el peso que le es atribuido a determinadas convicciones o «intereses» en función de los cuales se sanciona tanto al conocimiento producido, como la estrategia seguida para acceder a él. Es en torno a esta preocupación que cobran sentido postulados tales como, considerar que las grandes teorías con horizontes ambiciosos no pueden ser probadas de manera empírica o, por el contrario, posiciones donde la crítica se enfoca hacia los sesgos adquiridos por la intersubjetividad propia de los procesos sociales y educativos. Ambas posiciones, lejos de ajustarse a la dinámica de las nuevas redes de generación del saber, tienden a asimilarse progresivamente como una constante del quehacer de la investigación a través de los denominados procesos de «trasmisión de conocimiento» ${ }^{2}$, procesos que en consecuencia afectan los estilos de hacer investigación.

Además de contar con el respaldo de los mecanismos institucionales de trasmisión de conocimiento, el predominio de ciertos estilos para el desarrollo de las tareas de investigación se caracteriza por establecer parámetros que permiten estimar la visibilidad e impacto de los productos generados por la investigación. Así, la trasmisión de este tipo de patrones de una generación a otra queda básicamente asegurada por el arraigo de una experiencia científica más de carácter simulado que por la capacidad reflexiva y autocrítica de la comunidad que los adopta. Los rasgos específicos que distinguen a estas prácticas se reflejan en las formas en cómo al interior de las comunidades se establecen redes de comunicación, interacción e intercambio entre científicos-investigadores. Algunos de estos espacios se traducen en distintas formas reconocidas e institucionalizadas donde el encuentro de científicos e investigadores valida temas, perspectivas teóricas y metodológicas e incluso refrenda las vías para que el conocimiento por ellos producido sea sancionado y acreditado. Una de esas vías es el ejercicio de la citación, así como el estudio 
que la bibliometría ha hecho de la misma. La práctica de la citación y la acogida de que han sido objeto los resultados bibliométricos por parte de la comunidad académica, ha abierto un camino para estudiar la configuración de redes de conocimiento donde la dinámica de lo local-global está presente aun cuando su comportamiento es variable en el tiempo y en los distintos dominios disciplinarios, tema que es el abordado en este trabajo.

El objetivo de la presente investigación es el estudio particularizado a través de la citación de lo local-global presente en la dinámica de las redes de conocimiento construidas en los principales dominios disciplinarios de las ciencias sociales -economía, sociología, historia, antropología y educación-; este estudio es aplicado a un entorno regional específico sobre los productos de investigación —artículos en revistas - generados y publicados en y sobre Chiapas en un periodo que abarca ocho años (2000-2008). A diferencia de trabajos de investigación previamente realizados sobre el tema, donde el componente del contenido de los productos de investigación ocupó un papel central, este trabajo ofrece una visión de cómo se configura una red de conocimiento en un lapso de nueve años entre los investigadores que publican sobre la problemática social de Chiapas en cuatro de las revistas con respaldo institucional de mayor circulación en esta entidad.

La elección de trabajar sólo con los contenidos de las principales cuatro revistas ${ }^{3}$ reconocidas ampliamente por la comunidad de investigadores sociales por sobre todo lo publicado - tesis, libros, folletos, etc.-, responde a dos criterios: primero, de representatividad de contenido con respecto a los núcleos temáticos, las metodologías y los usos teórico-conceptuales predominantes a lo largo de las tres últimas décadas; segundo, el respaldo institucional de las publicaciones, ya que ninguna de las cuatro revistas-anuarios de investigación existentes cumplen con los parámetros de consistencia acordados por la mayor parte de las comunidades nacionales e internacionales para reconocer el peso e impacto de una revista científica; requerimientos que tienen que ver principalmente con su puntualidad y frecuencia de publicación, así como con la composición de sus respectivos órganos editoriales. ${ }^{4}$ Así, el corpus de artículos o productos de investigación social 
incluidos y revisados en este trabajo representa una muestra más que representativa —entendida ésta no en términos estrictamente estadísticos - acerca del predominio de determinadas miradas cuya filiación disciplinaria, teórica y metodológica, da cuenta de una larga tradición, hoy institucionalizada, sobre determinadas concepciones y prácticas de investigación sobre la problemática regional.

El interés es valorar hasta qué punto la dinámica y el comportamiento de las redes de conocimiento conformadas en los dominios disciplinarios de las ciencias sociales a través de la práctica de la citación, pueden ser consideradas como un instrumento para evaluar el potencial de una comunidad académica para insertarse en los actuales procesos de generación de saber y, con ello, re-posicionarse en el nuevo espacio local-global. Este análisis permite entender cómo se desarrollan los fenómenos de comunicación y de circulación de las ideas entre las comunidades académicas, así como también estimar su eventual impacto en el contexto de la internacionalización de las ciencias sociales.

\section{LOS INDICADORES BIBLIOMÉTRICOS COMO FUNDAMENTO PARA EL ESTUDIO DE LAS REDES DE CONOCIMIENTO}

Han sido los estudios realizados por la bibliometría los que de manera sistemática han ofrecido pistas importantes acerca de cómo se configuran las redes de conocimiento en una comunidad científica. La bibliometría ${ }^{5}$ se define como «...la aplicación de los métodos estadísticos y matemáticos dispuestos para definir los procesos de la comunicación escrita y la naturaleza y el desarrollo de las disciplinas científicas mediante técnicas de recuento y análisis de dicha comunicación» (Pritchard 1969: 348-349). Entre las principales técnicas bibliométricas figuran: el conteo de artículos científicos publicados durante un periodo determinado con el fin de estimar la producción de un conjunto o de un subconjunto del sistema científico, pudiéndose con ello establecer también la intensidad de la producción en un determinado dominio - especialización —; la contabilidad de citas hechas a una revista con la finalidad de medir el impacto científico de la investigación y, por último, la cartografía que permite acercarse a la evolución de campos de especialidad emergentes en función del tiempo; en este último caso, el análisis se sustenta en la concurrencia y 
coincidencia de palabras clave, de citas y de referencias bibliográficas.

De acuerdo con Science Metrix, la bibliometría tiene como propósito medir la actividad científica generando estadísticas sobre los artículos científicos reunidos en las grandes bases de datos, convirtiéndose en una herramienta flexible que permite: estudiar los fenómenos sociológicos vinculados a las comunidades científicas, establecer una vigilancia científica/estratégica, técnica, tecnológica o competitiva, efectuar y administrar programas de investigación, así como también evaluar la actividad de investigación.

La utilización de los métodos bibliométricos de alcance internacional en los dominios de las ciencias sociales y la educación — tales como la base de datos Thompson/ISI y SCOPUS/Elsevier-, plantean dos grandes problemas que autores como Yves Gingras y Philippe Jeannin han reiteradamente señalado. Por un lado, la ausencia en estas bases de una cobertura amplia de publicaciones que no se restrinja sólo a los artículos incluidos en revistas, y por otro la limitada producción científica no incluida, ya que no se encuentra publicada en inglés. A pesar de tales inconvenientes, los criterios utilizados por los grandes bancos de información pueden ser considerados no tanto para «evaluar la productividad» de una determinada comunidad sino más bien para estudiar la evolución de las prácticas de investigación, identificando tanto las características de los artículos en sí mismos como también las referencias bibliográficas que ellos contienen. En el primer caso, será posible tener una idea de la tendencia a firmar los artículos con varios autores ya sea en colaboración local, nacional o internacional; en el segundo caso, será posible apreciar la tendencia en los distintos dominios disciplinarios y temáticos a utilizar referencias locales, nacionales o internacionales ya sea de orden teórico o temático.

Cualquiera que fuese el propósito establecido para hacer uso de las bases de datos existentes sobre las publicaciones científicas, el manejo de estas últimas queda sujeto a la pertinencia que pudiese existir entre los criterios de construcción de los indicadores contenidos en los respectivos bancos de información, y aquellos propios de los procesos a 
lo cuales se pretende dar respuesta. Un indicador construido con la finalidad de dar cuenta de aspectos aislados de un fenómeno difícilmente contará con el alcance explicativo para dar respuesta a problemáticas complejas cuyo comportamiento en la realidad se modifica constantemente. Así, el registro del número de publicaciones generadas por un investigador o bien por uno o varios dominios disciplinarios, considerando incluso la sistematización de los datos bibliográficos y el origen de la edición de tales publicaciones, si bien resultan insuficientes para dar cuenta de la dinámica del campo científico y de las prácticas de investigación, son elementos que permiten establecer los flujos de comunicación establecidos por los investigadores-autores con respecto a las redes de las cuales forman parte.

Las propiedades que debe guardar la construcción de un indicador se encuentran estrechamente vinculadas con la naturaleza del objeto de estudio. Gingras (2008: 5-8, 13) señala que tales propiedades son tres.

- La primera propiedad de un buen indicador yace en su adecuación al objeto, condición que en el ámbito de las ciencias sociales exige la realización de pruebas, así como el análisis de los modos de producción de saberes vigentes en los distintos dominios disciplinarios.

- Una segunda propiedad de un buen indicador es que debe ser homogéneo en su composición, por ejemplo, un indicador de la intensidad de la actividad científica a escala nacional es el ofrecido por el número de publicaciones registradas en las principales revistas científicas y el valor de la inversión en la investigación; se trata de una cualidad que permite estimaciones más precisas sobre la intensidad de la actividad científica con alcances incluso de tipo comparativo - entre varios dominios disciplinarios, regiones o países - bajo el criterio de la eficiencia. La construcción de indicadores simples y homogéneos permite interpretarlos más fácilmente y apreciar si ellos cambian de manera continua o abrupta; asimismo, la fluctuación registrada en un solo rango de un año a otro se deberá seguramente a la 
variación natural de los procesos estudiados sin que ello deba dar lugar a una sobre interpretación de los datos registrados. Dicha cualidad deja de cumplirse si este mismo indicador es considerado como medida o parámetro de calidad. En este caso, su composición pasa a ser heterogénea variando de manera imprevista y sin contar con un significado claro; es cuando se pretende medir la productividad de una comunidad científica por el número de publicaciones reportadas.

- Por último, la tercera propiedad con la que un buen indicador debe contar es que éste debe variar de acuerdo con la inercia propia del objeto medido. Algunos fenómenos varían más o menos rápidamente según sus características intrínsecas; un ejemplo puede ser la intención de medir el impacto social de la investigación en el terreno de la historia, a diferencia del que pueda registrar la investigación para el desarrollo en economía.

La adecuada construcción de indicadores debe también atender a criterios claros de interpretación, manteniéndose en este sentido dentro de los límites de su validez; esta vigilancia garantiza su correspondencia con las condiciones que guardan en la realidad los fenómenos estudiados. Lejos de pretender evaluar el desempeño individual de un investigador, el diseño de indicadores para conocer la evolución y trasformación de las prácticas de investigación en un entorno regional específico está encaminado a la identificación de las redes de conocimiento que median entre el trabajo individual y las tendencias de la práctica de la investigación en los diversos dominios disciplinarios.

Las redes de conocimiento conformadas por la bibliografía referenciada o citada en los artículos publicados en revistas no se limitan a la identificación y sistematización de documentos —objetivo bibliográfico - sino al acercamiento sociológico de las elecciones de estudio efectuadas por los autores o investigadores sobre el conocimiento por ellos producido, difundido y objetivado a través de sus publicaciones. En la conformación de redes de conocimiento intervienen principalmente dos aspectos: a) el tipo de tradición científica prevaleciente en una determinada comunidad en un periodo determinado y su 
posición ocupada en el contexto del desarrollo de las ciencias sociales, y b) el impacto social estimado a partir de los elementos cognitivos provenientes del entorno histórico-social e institucional asimilados a la experiencia de dicha comunidad, tanto en su condición de actores sociales como de productores de conocimiento. ${ }^{6}$

\section{LAS REDES DE CONOCIMIENTO PRESENTES EN LAS PRÁCTICAS DE CITACIÓN}

Los estudios de redes sociales se centran en la identificación de estructuras a partir del análisis de las relaciones existentes entre determinados elementos -instituciones, temas u objetos de estudio, metodologías y referencias teóricas y temáticas - independientemente de los atributos o características de esos elementos por separado. Este estudio asume que esas estructuras ejercen algún tipo de influencia en el comportamiento de los elementos que componen el sistema (Molina, Muñoz y Losego 2009), dando como resultado conformaciones — desde el punto de vista disciplinario y temático- de redes de comunicación particulares, las tradiciones y las condiciones de origen de los trabajos publicados. La distribución disciplinaria de los trabajos producidos, las estrategias metodológicas adoptadas, las preferencias teóricas y temáticas, así como las redes construidas por cada uno de los autores, son elementos que ofrecen la posibilidad de conocer primero, la estructura y la dinámica de las redes sociales establecidas por una comunidad académica en el contexto de las sociedades en desarrollo con respecto a la innovación en el conocimiento local; y segundo, en qué medida se establecen vínculos entre la investigación social del país y la realizada en otros entornos nacionales y culturales. 
Cuadro 1. Número de artículos publicado por disciplina

\begin{tabular}{|c|c|c|c|c|c|}
\hline Disciplina & $N^{o}$ & \multicolumn{4}{|c|}{ Publicación } \\
\hline & & $\begin{array}{l}\text { Anuario } \\
\text { Cesmeca }\end{array}$ & Anuario IEI & LiminaR & $\begin{array}{l}\text { Pueblos y } \\
\text { fronteras }\end{array}$ \\
\hline Antropología & 81 & 27 & 33 & 12 & 9 \\
\hline Sociología & 43 & 20 & 6 & 15 & 2 \\
\hline Historia & 23 & 8 & 7 & 4 & 4 \\
\hline Arqueología & 14 & 6 & 0 & 3 & 5 \\
\hline $\begin{array}{l}\text { Desarrollo } \\
\text { económico }\end{array}$ & 8 & 3 & 0 & 1 & 4 \\
\hline $\begin{array}{l}\text { Psicología } \\
\text { educativa }\end{array}$ & 2 & 2 & 0 & 0 & 0 \\
\hline Informe técnico & 1 & 1 & 0 & 0 & 0 \\
\hline Total de artículos & 172 & 67 & 46 & 35 & 24 \\
\hline
\end{tabular}

Fuente: Elaboración propia.

Las cuatro publicaciones periódicas consideradas en este estudio concentran un total de 172 artículos pertenecientes a los campos disciplinarios propios de las ciencias sociales y que además abordan de manera directa y explicita alguna problemática social, cultural, política y económica de Chiapas. Destacan las dos publicaciones del Centro de Estudios Superiores de México y Centroamérica (Cesmeca-Unicach): el anuario del Cesmeca y la revista Liminar, ${ }^{7}$ ya que concentran cerca de $60 \%$ del total de los artículos estudiados, son seguidas por los contenidos en el anuario del Instituto del Estudios Indígenas (IEI-Unach) con 27\%, y por la revista Pueblos y fronteras del Programa de Investigaciones Multidisciplinarias sobre Mesoámerica y el Sureste de la Universidad Nacional Autónoma de México (Proiimse-UNAM) con cerca de $14 \%$.

La inclinación hacia los estudios antropológicos es característica de las tres revistas aun cuando en el caso del Anuario de IEI esta inclinación es prácticamente predominante, dejando fuera del centro de atención los abordajes provenientes de otros campos disciplinarios. En contraste, el comportamiento que en conjunto muestran los trabajos publicados por Liminar y Pueblos y fronteras con respecto a la atención multidisciplinaria sobre la problemática social de Chiapas, resulta más equilibrado, incluso cuando el número 
de artículos reportados sea reducido para el periodo estudiado.

El predomino de la Antropología como acercamiento disciplinario a la problemática social se ha mantenido por décadas en las instituciones universitarias y de investigación social de Chiapas, principalmente aquellas concentradas geográficamente en la ciudad de San Cristóbal de Las Casas. El avance de la sociología, aunque muy ligada a temas de desarrollo económico, ocupa un segundo lugar en la atención de investigadores. Las aproximaciones efectuadas desde las perspectivas histórica, historiográfica y la archivística son también, aunque en menor proporción, afines a la comunidad de investigadores locales; su principal característica es el apego que guardan con sus respectivas tradiciones en cuanto al uso e interpretación de las fuentes. El tratamiento predominantemente técnico realizado por la Arqueología hace de los trabajos publicados en este campo un material difícilmente incorporable al resto de las ciencias sociales, estableciendo así sus vínculos más con la experiencia historiográfica que con otros ámbitos teóricos de la historia.

Cuadro 2. Artículos que contienen referencias teóricas y/o temáticas

\begin{tabular}{|c|c|c|c|c|c|c|c|c|}
\hline \multirow[t]{2}{*}{ Disciplina } & \multirow[t]{2}{*}{$N^{\circ}$} & \multirow{2}{*}{$\begin{array}{c}\text { Sólo } \\
\text { teóricas } \\
\end{array}$} & \multirow{2}{*}{$\begin{array}{c}\text { Sólo } \\
\text { temáticas } \\
\end{array}$} & \multicolumn{5}{|c|}{ Teóricas y temáticas } \\
\hline & & & & $\begin{array}{c}\text { A. } \\
\text { Cesmeca }\end{array}$ & $\begin{array}{l}\text { A. } \\
\text { IEI }\end{array}$ & Liminar & $\mathrm{P}$ y $\mathrm{F}$ & Total \\
\hline Antropología & 81 & 1 & 42 & 17 & 4 & 7 & 5 & 33 \\
\hline Sociología & 43 & 1 & 22 & 10 & 2 & 4 & 0 & 16 \\
\hline Historia & 23 & 0 & 20 & 0 & 3 & 0 & 0 & 3 \\
\hline Arqueología & 14 & 0 & 14 & 0 & 0 & 0 & 0 & 0 \\
\hline $\begin{array}{l}\text { Desarrollo } \\
\text { económico }\end{array}$ & 8 & 1 & 4 & 1 & 0 & 1 & 1 & 3 \\
\hline $\begin{array}{l}\text { Psicología } \\
\text { educativa }\end{array}$ & 2 & 1 & 1 & 0 & 0 & 0 & 0 & 0 \\
\hline $\begin{array}{l}\text { Informe } \\
\text { técnico }\end{array}$ & 1 & 0 & 0 & 0 & 0 & 0 & 0 & 0 \\
\hline $\begin{array}{c}\text { Total } \\
\text { artículos }\end{array}$ & 172 & 4 & 102 & 28 & 9 & 12 & 6 & 55 \\
\hline
\end{tabular}

Fuente: Elaboración propia. 
El predominio de la referencia temática sobre la teórica es un rasgo característico de los trabajos publicados en los diferentes campos disciplinarios. Sólo una tercera parte del total de los trabajos muestran un mayor apego a la práctica de construcción teórica de los objetos de estudio, ahí donde la referencia al pensamiento teórico queda establecida, a juicio del autor, por la naturaleza del referente empírico. Más de las dos terceras partes de estos trabajos se encuentran publicados por el anuario del Cesmeca y por la revista Liminar, destacando los realizados desde las ópticas antropológica y sociológica. Por el contrario, los trabajos circunscritos en el campo de la historia y de la psicología se caracterizan por prescindir de toda argumentación teórica como plataforma para el abordaje de sus respectivos objetos de estudio. Cabe señalar que el uso de la referencia teórica y temática no asegura una óptima construcción de los objetos de estudio abordados por todos los trabajos aquí considerados y que reúnen esta característica. ${ }^{8}$

El predominio de las referencias temáticas como particularidad de las otras dos terceras partes de los trabajos se presenta en una proporción similar para la Antropología, la Sociología y el Desarrollo económico, no siendo así para los trabajos realizados desde las perspectivas histórica y arqueológica cuyo soporte es fundamentalmente la referencia temática. La presencia de referencias temáticas no limita el alcance heurístico e innovador de los resultados logrados en todos los trabajos. ${ }^{9}$ 
Cuadro 3. Autores más citados como referencia teórica en las distintas disciplinas

\begin{tabular}{|c|c|c|c|c|}
\hline Autor & $N^{\circ}$ & Disciplina & Citado por: & $\begin{array}{l}\text { Revista } \\
\text {-frecuencia- }\end{array}$ \\
\hline $\begin{array}{l}\text { Pierre } \\
\text { Bourdieu }\end{array}$ & 18 & $\begin{array}{l}\text { Antropología (9); } \\
\text { Sociología (2); } \\
\text { Antropología } \\
\text { política; } \\
\text { Antropología } \\
\text { (género) (2); } \\
\text { Antropología } \\
\text { cultural; } \\
\text { Antropología y } \\
\text { etnografía (2); } \\
\text { Sociología jurídica. }\end{array}$ & $\begin{array}{l}\text { Imberton; García Méndez; } \\
\text { Hvostoff; Ruiz Castillo; } \\
\text { Oliveira Bustamante; Ramos } \\
\text { Maza; Escalona Victoria; De } \\
\text { la Cruz López; Page Pliego; } \\
\text { Lisbona Guillén; Pinto Durán; } \\
\text { Fernández Zamora; Agudo } \\
\text { Sanchiz; Sulca Báez; Oliveira } \\
\text { y Sánchez; Martínez Martínez; } \\
\text { Paniagua Mijangos; Ramírez } \\
\text { Poloche }\end{array}$ & $\begin{array}{l}\text { Anuario } \\
\text { Cesmeca (5); } \\
\text { Anuario IEI (4); } \\
\text { Liminar (4); } \\
\text { Pueblos y } \\
\text { fronteras (5) }\end{array}$ \\
\hline $\begin{array}{l}\text { Max } \\
\text { Weber }\end{array}$ & 7 & $\begin{array}{l}\text { Antropología } \\
\text { social; Sociología } \\
\text { política (2); } \\
\text { Antropología } \\
\text { política; } \\
\text { Antropología (2); } \\
\text { Desarrollo } \\
\text { económico } \\
\end{array}$ & $\begin{array}{l}\text { Del Carpio Penagos; } \\
\text { Rodríguez Castillo (2); } \\
\text { Escalona Victoria; Lisbona } \\
\text { Guillén (2); Márquez Rosado }\end{array}$ & $\begin{array}{l}\text { Anuario } \\
\text { Cesmeca (3); } \\
\text { Liminar (2); } \\
\text { Pueblos y } \\
\text { fronteras (2) }\end{array}$ \\
\hline $\begin{array}{l}\text { Giovanni } \\
\text { Sartori } \\
\end{array}$ & 4 & $\begin{array}{l}\text { Sociología política } \\
\text { (3); Sociología }\end{array}$ & $\begin{array}{l}\text { García Aguilar (2); Ascencio } \\
\text { Franco; Hernández Molina }\end{array}$ & $\begin{array}{l}\text { Anuario } \\
\text { Cesmeca (4) }\end{array}$ \\
\hline $\begin{array}{l}\text { Clifford } \\
\text { Geertz }\end{array}$ & 3 & Antropología (3) & $\begin{array}{l}\text { Schenerock; Page Pliego; } \\
\text { Paniagua Mijangos }\end{array}$ & $\begin{array}{l}\text { Liminar; } \\
\text { Pueblos y } \\
\text { fronteras (2) }\end{array}$ \\
\hline $\begin{array}{l}\text { Maurice } \\
\text { Godelier }\end{array}$ & 3 & $\begin{array}{l}\text { Antropología; } \\
\text { Desarrollo } \\
\text { económico; } \\
\text { Antropología y } \\
\text { Etnografía. }\end{array}$ & $\begin{array}{l}\text { De la Cruz López; Márquez } \\
\text { Rosado; Pinto Durán }\end{array}$ & $\begin{array}{l}\text { Pueblos y } \\
\text { fronteras (3) }\end{array}$ \\
\hline $\begin{array}{l}\text { Norberto } \\
\text { Bobbio } \\
\end{array}$ & 3 & $\begin{array}{l}\text { Sociología política } \\
\text { (3) }\end{array}$ & $\begin{array}{l}\text { García Aguilar; Burguete Cal } \\
\text { y Mayor; Hernández Molina. }\end{array}$ & $\begin{array}{l}\text { Anuario } \\
\text { Cesmeca (3) }\end{array}$ \\
\hline $\begin{array}{l}\text { Marc } \\
\text { Augé }\end{array}$ & 3 & Antropología (3) & $\begin{array}{l}\text { Page Pliego; Lisbona Guillén; } \\
\text { Paniagua Mijangos }\end{array}$ & $\begin{array}{l}\text { Anuario } \\
\text { Cesmeca; } \\
\text { Pueblos y } \\
\text { fronteras (2) }\end{array}$ \\
\hline $\begin{array}{l}\text { Guillermo } \\
\text { Bonfil }\end{array}$ & 2 & $\begin{array}{l}\text { Antropología; } \\
\text { Sociología }\end{array}$ & $\begin{array}{l}\text { Paniagua Mijangos; Sulca } \\
\text { Báez }\end{array}$ & $\begin{array}{l}\text { Anuario IEI; } \\
\text { Anuario } \\
\text { Cesmeca }\end{array}$ \\
\hline
\end{tabular}

Fuente: Elaboración propia.

La incidencia del pensamiento de los teóricos sociales es escasa y además difiere por cada disciplina. No obstante, una constante aunque poco representativa pero que figura en todas 
las disciplinas es la obra de Pierre Bourdieu, presente de manera más clara en la investigación antropológica en la región. En mucho menor medida se consigna la referencia a Max Weber y a otros autores más ligados a los campos de la Antropología y la Sociología. Aunque muy reducida, la presencia de la referencia teórica recae proporcionalmente en prácticamente todas las revistas estudiadas, salvo el caso del anuario del IEI, donde ésta es prácticamente inexistente.

Cuadro 4. Autores más citados como referencia temática

\begin{tabular}{|c|c|c|c|c|}
\hline $\begin{array}{l}\text { Autor } \\
\text { citado }\end{array}$ & Citado por: & $\begin{array}{c}\text { Disciplina desde la } \\
\text { que es citado: }\end{array}$ & $\begin{array}{c}\text { Revistas de } \\
\text { referencia donde } \\
\text { es citado }\end{array}$ & $\begin{array}{c}\text { Tot. de } \\
\text { referencias }\end{array}$ \\
\hline $\begin{array}{l}\text { Mario } \\
\text { Humberto } \\
\text { Ruz }\end{array}$ & $\begin{array}{l}\text { Cosamalón Aguilar (2); } \\
\text { Pinto Durán (2); } \\
\text { Sonnlentier; Toledo Tello; } \\
\text { Gómez Hernández; } \\
\text { Fernández Galán; Aramoni } \\
\text { Calderón (3); Solís Cruz; } \\
\text { Pinto y López; Gómez } \\
\text { Hernández; Viqueira; } \\
\text { Escalona Victoria; Gutiérrez } \\
\text { Alfonso; Rivera Farfán; } \\
\text { López Jiménez; Tovalín } \\
\text { Ahumada; Guzmán } \\
\text { Coronado; Palomo Infante; } \\
\text { Gussinyer y García; } \\
\text { Mendoza García; Brown; } \\
\text { Gómez y Escalona; } \\
\text { Imberton; Ramos Maza; } \\
\text { Vázquez Olivera; Estrada } \\
\text { Saavedra; Anguiano Téllez; } \\
\text { Moreno Heras; Gómez y } \\
\text { Pinto; Ramírez Poloche; } \\
\text { Castañeda Seijas; Burguete } \\
\text { Cal y Mayor }\end{array}$ & $\begin{array}{l}\text { Historia social (3); } \\
\text { Historia (7); } \\
\text { Antropología } \\
\text { cultural (3); } \\
\text { Antropología y } \\
\text { Etnografía (3); } \\
\text { Sociología política } \\
\text { (2); Antropología } \\
\text { (13); Antropología } \\
\text { política; } \\
\text { Arqueología (2); } \\
\text { Sociología; Historia } \\
\text { económica-social }\end{array}$ & $\begin{array}{l}\text { Anuario } \\
\text { Cesmeca; } \\
\text { Anuario IEI; } \\
\text { Liminar; Pueblos } \\
\text { y fronteras }\end{array}$ & 36 \\
\hline $\begin{array}{l}\text { Juan Pedro } \\
\text { Viqueira }\end{array}$ & $\begin{array}{l}\text { García Aguilar; Sonnlentier; } \\
\text { Toledo Tello; Fernández } \\
\text { Galán; Hernández Castillo; } \\
\text { Escalona Victoria (2); } \\
\text { Trench; Lisbona Guillén (2); } \\
\text { Pinto Durán; Mendoza } \\
\text { García; Ascencio Franco; } \\
\text { Aramoni Calderón; Garza } \\
\text { Caligaris; Ramos Maza; } \\
\text { Vázquez Olivera; Castro }\end{array}$ & $\begin{array}{l}\text { Sociología política } \\
\text { (5); Antropología; } \\
\text { Historia (4); } \\
\text { Antropología y } \\
\text { Etnografía (3); } \\
\text { Antropología (5); } \\
\text { Antropología } \\
\text { cultural; Historia } \\
\text { social }\end{array}$ & $\begin{array}{l}\text { Anuario } \\
\text { Cesmeca; } \\
\text { Anuario IEI; } \\
\text { Liminar; Pueblos } \\
\text { y Fronteras }\end{array}$ & 20 \\
\hline
\end{tabular}




\begin{tabular}{|c|c|c|c|c|}
\hline & $\begin{array}{l}\text { Apreza; López y Pérez; } \\
\text { Ramírez Poloche. }\end{array}$ & & & \\
\hline De Vos Jan & $\begin{array}{l}\text { Fernández Galán; Viqueira; } \\
\text { Trench; Escalona Victoria; } \\
\text { Rivera Farfán (2); Palomo } \\
\text { Infante; Pinto Durán; Garza } \\
\text { Caligaris; López Reyes; } \\
\text { Trench y Kölher; Gutiérrez } \\
\text { Cruz; Vázquez Olivera; } \\
\text { Carrasco, Benítez y } \\
\text { Rodríguez; Roblero } \\
\text { Morales; Castañeda Seijas; } \\
\text { Morales Barragán }\end{array}$ & $\begin{array}{l}\text { Historia; } \\
\text { Antropología (7); } \\
\text { Historia (3); } \\
\text { Antropología y } \\
\text { Etnografía; } \\
\text { Sociología; Historia } \\
\text { social; Sociología } \\
\text { política; } \\
\text { Antropología y } \\
\text { medio ambiente; } \\
\text { Desarrollo } \\
\text { económico }\end{array}$ & $\begin{array}{l}\text { Anuario IEI; } \\
\text { Liminar; Pueblos } \\
\text { y fronteras; } \\
\text { Anuario Cesmeca }\end{array}$ & 17 \\
\hline $\begin{array}{l}\text { Daniel } \\
\text { Villafuerte }\end{array}$ & $\begin{array}{l}\text { Ascencio Franco; García } \\
\text { Aguilar; Solís Cruz; } \\
\text { Villafuerte Solís (3); } \\
\text { Villafuerte y García (2); } \\
\text { Angulo Barredo; Escalona } \\
\text { Victoria; Rivera Farfán (2); } \\
\text { Márquez Rosado; Montoya } \\
\text { y Mijangos; Lisbona } \\
\text { Guillén; Agudo Sanchiz; } \\
\text { Sulca Báez; Ramos Maza; } \\
\text { García y Rodríguez; Pérez } \\
\text { Roblero; Anguiano Téllez }\end{array}$ & $\begin{array}{l}\text { Antropología social; } \\
\text { Antropología } \\
\text { cultural; Sociología } \\
\text { política (2); } \\
\text { Antropología (7); } \\
\text { Sociología (8); } \\
\text { Desarrollo } \\
\text { económico (2) }\end{array}$ & $\begin{array}{l}\text { Anuario } \\
\text { Cesmeca; } \\
\text { Liminar; Anuario } \\
\text { IEI; Pueblos y } \\
\text { Fronteras }\end{array}$ & $22-5=17$ \\
\hline $\begin{array}{l}\text { George } \\
\text { Collier }\end{array}$ & $\begin{array}{l}\text { Garza Caligaris; Pinto y } \\
\text { López; Bigmore y } \\
\text { Perezgrovas; Toledo y } \\
\text { Garza; Kampwrith; } \\
\text { Rodríguez Castillo; } \\
\text { Paniagua Mijangos; Brown; } \\
\text { Cardús I Font; Castro } \\
\text { Apreza; Carrasco, Benítez y } \\
\text { Rodríguez; Paniagua } \\
\text { Mijangos; Moreno Heras }\end{array}$ & $\begin{array}{l}\text { Historia; } \\
\text { Antropología } \\
\text { política; } \\
\text { Antropología (5); } \\
\text { Sociología; } \\
\text { Sociología política } \\
\text { (3); Historia; } \\
\text { Historia económica } \\
\text { y social. }\end{array}$ & $\begin{array}{l}\text { Anuario IEI; } \\
\text { Liminar; Anuario } \\
\text { Cesmeca; } \\
\text { Pueblos y } \\
\text { fronteras }\end{array}$ & 13 \\
\hline Jan Rus & $\begin{array}{l}\text { Avella Alamitos; Jan Rus } \\
\text { (2); Sonnlentier; Garza } \\
\text { Caligaris; Olivera } \\
\text { Bustamente; Escalona } \\
\text { Victoria (2); Hernández } \\
\text { Castillo; Paniagua Mijangos } \\
\text { (2); Castro Apreza; Ortiz } \\
\text { Herrera; Moreno Heras }\end{array}$ & $\begin{array}{l}\text { Historia social; } \\
\text { Antropología } \\
\text { cultural; Sociología } \\
\text { política (2); } \\
\text { Antropología (5); } \\
\text { Historia (2); } \\
\text { Antropología } \\
\text { género; } \\
\text { Antropología y } \\
\text { Etnografía; Historia } \\
\text { económica y social } \\
\end{array}$ & $\begin{array}{l}\text { Anuario Cesmeca } \\
\text { (2); Anuario IEI; } \\
\text { Liminar; Pueblos } \\
\text { y fronteras }\end{array}$ & $14-2=12$ \\
\hline
\end{tabular}




\begin{tabular}{|c|c|c|c|c|}
\hline $\begin{array}{l}\text { Dolores } \\
\text { Aramoni }\end{array}$ & $\begin{array}{l}\text { García Méndez; Venturoli; } \\
\text { Domenichi y Lee; } \\
\text { Fernández Galán; López } \\
\text { Espinoza; Viqueira; } \\
\text { Hernández Castillo; Lee } \\
\text { Witting; Rivera Farfán; } \\
\text { Palomo Infante; Aramoni } \\
\text { Calderón }\end{array}$ & $\begin{array}{l}\text { Antropología (4); } \\
\text { Antropología } \\
\text { cultural y } \\
\text { Arqueología; } \\
\text { Arqueología (2); } \\
\text { Historia (3); } \\
\text { Antropología y } \\
\text { etnografía }\end{array}$ & $\begin{array}{l}\text { Anuario } \\
\text { Cesmeca; } \\
\text { Anuario IEI; } \\
\text { Liminar; Pueblos } \\
\text { y fronteras }\end{array}$ & $11-1=10$ \\
\hline $\begin{array}{l}\text { Thomas } \\
\text { Benjamin }\end{array}$ & $\begin{array}{l}\text { Avella Alamitos; Toledo } \\
\text { Tello; Kampwrith; Rivera } \\
\text { Farfán; Lisbona Guillén; } \\
\text { Pinto Durán; Brown; López } \\
\text { Reyes: Agudo Sanchiz; Del } \\
\text { Carpio Penagos }\end{array}$ & $\begin{array}{l}\text { Historia social; } \\
\text { Antropología (6); } \\
\text { Historia (2); } \\
\text { Antropología y } \\
\text { Etnografía }\end{array}$ & $\begin{array}{l}\text { Anuario } \\
\text { Cesmeca; } \\
\text { Anuario IEI; } \\
\text { Pueblos y } \\
\text { fronteras }\end{array}$ & 10 \\
\hline $\begin{array}{l}\text { Carlos } \\
\text { Navarrete }\end{array}$ & $\begin{array}{l}\text { Domenici y Lee; Fernández } \\
\text { Galán; Gutiérrez Alfonso; } \\
\text { Rivera Farfán; Linares y } \\
\text { Silva; Linares Villanueva; } \\
\text { Lee Whitng; López Reyes; } \\
\text { Esponda Jimeno }\end{array}$ & $\begin{array}{l}\text { Arqueología; } \\
\text { Historia (2); } \\
\text { Antropología (5) }\end{array}$ & $\begin{array}{l}\text { Anuario } \\
\text { Cesmeca; } \\
\text { Anuario IEI; } \\
\text { Pueblos y } \\
\text { fronteras }\end{array}$ & 9 \\
\hline Neil Harvey & $\begin{array}{l}\text { García Aguilar; Kampwrith; } \\
\text { Escalona Victoria; Agudo } \\
\text { Sanchiz; Estrada Saavedra; } \\
\text { Castro Apreza; Carrasco, } \\
\text { Benítez y Rodríguez; } \\
\text { Hernández Molina }\end{array}$ & $\begin{array}{l}\text { Sociología política } \\
\text { (4); Antropología } \\
\text { (3); Historia social }\end{array}$ & $\begin{array}{l}\text { Anuario IEI; } \\
\text { Liminar; Anuario } \\
\text { Cesmeca }\end{array}$ & 8 \\
\hline $\begin{array}{l}\text { Xóchitl } \\
\text { Leyva }\end{array}$ & $\begin{array}{l}\text { Kohler y Trench; Rodríguez } \\
\text { Castillo (2); Hernández } \\
\text { Castillo; Lisbona Guillén; } \\
\text { Gerber; Castro Apreza }\end{array}$ & $\begin{array}{l}\text { Antropología } \\
\text { cultural y Medios } \\
\text { de comunicación; } \\
\text { Antropología } \\
\text { política; } \\
\text { Antropología y } \\
\text { Etnografía; } \\
\text { Antropología (2); } \\
\text { Sociología política } \\
\text { (2) }\end{array}$ & $\begin{array}{l}\text { Anuario } \\
\text { Cesmeca; } \\
\text { Liminar; Anuario } \\
\text { IEI }\end{array}$ & 7 \\
\hline $\begin{array}{l}\text { Miguel } \\
\text { Lisbona } \\
\text { Guillén }\end{array}$ & $\begin{array}{l}\text { Lisbona Guillén (4); Del } \\
\text { Carpio Penagos; Pinto } \\
\text { Durán; Villafuerte Solís; } \\
\text { Viqueira; Rivera Farfán; } \\
\text { Aramoni Calderón; } \\
\text { Castañeda Seíjas }\end{array}$ & $\begin{array}{l}\text { Antropología social } \\
\text { (2); Antropología } \\
\text { política; Sociología; } \\
\text { Antropología (3); } \\
\text { historia }\end{array}$ & $\begin{array}{l}\text { Anuario } \\
\text { Cesmeca; } \\
\text { Liminar; Anuario } \\
\text { IEI; Pueblos y } \\
\text { Fronteras }\end{array}$ & $11-4=7$ \\
\hline $\begin{array}{l}\text { Graciela } \\
\text { Freyermuth }\end{array}$ & $\begin{array}{l}\text { Freyermuth; Pinto Durán y } \\
\text { López Moya; Kampwrith; } \\
\text { Hernández Castillo; Castro } \\
\text { Apreza; González Figueroa. }\end{array}$ & $\begin{array}{l}\text { Antropología (2); } \\
\text { Antropología } \\
\text { política; } \\
\text { Antropología y }\end{array}$ & $\begin{array}{l}\text { Anuario } \\
\text { Cesmeca; } \\
\text { Liminar; Anuario } \\
\text { IEI; }\end{array}$ & 6 \\
\hline
\end{tabular}




\begin{tabular}{|l|l|l|l|c|}
\hline & & $\begin{array}{l}\text { Etnografía; } \\
\text { Sociología (2) }\end{array}$ & \\
\hline Henri Favre & $\begin{array}{l}\text { Page Pliego; Escalona } \\
\text { Victoria (2); Paniagua } \\
\text { Miñangos (2); Moreno } \\
\text { Heras }\end{array}$ & $\begin{array}{l}\text { Antropología (5); } \\
\text { Antropología social }\end{array}$ & $\begin{array}{l}\text { Anuario } \\
\text { Cesmeca; } \\
\text { Anuario IEI; } \\
\text { Liminar; Pueblos } \\
\text { y fronteras }\end{array}$ & 6 \\
\hline $\begin{array}{l}\text { Guillermo } \\
\text { Bonfil }\end{array}$ & $\begin{array}{l}\text { Hvostoff; Hernández } \\
\text { Castillo; Escalona Victoria; } \\
\text { Palomo Infante; Agudo } \\
\text { Sanchiz }\end{array}$ & $\begin{array}{l}\text { Sociología; } \\
\text { Antropología y } \\
\text { etnografía; } \\
\text { Antropología (2); } \\
\text { Historia }\end{array}$ & $\begin{array}{l}\text { Liminar; Pueblos } \\
\text { y fronteras }\end{array}$ & 5 \\
\hline $\begin{array}{l}\text { Ascencio } \\
\text { Franco }\end{array}$ & $\begin{array}{l}\text { Rodríguez Castillo; } \\
\text { Kampwrith; Rivera Farfán; } \\
\text { Carrasco, Benítez y } \\
\text { Rodríguez; Burguete Cal y } \\
\text { Mayor }\end{array}$ & $\begin{array}{l}\text { Antropología } \\
\text { política; } \\
\text { Antropología (2); } \\
\text { Sociología política } \\
(2)\end{array}$ & $\begin{array}{l}\text { Liminar; Anuario } \\
\text { IEI; Anuario } \\
\text { Cesmeca; Pueblos } \\
\text { y fronteras }\end{array}$ & 5 \\
\hline
\end{tabular}

Fuente: Elaboración propia.

Aun cuando la referencia temática es dominante en los trabajos publicados por todas las revistas estudiadas, su dispersión entre las disciplinas consideradas da lugar a un peculiar comportamiento de las mismas; es decir, las referencias efectuadas obedecen más al grado de penetración y de circulación local del trabajo de un autor que a su filiación teórica y/o a sus aportes al campo disciplinario global de origen. Es el caso de los trabajos de Mario Humberto Ruz, Juan Pedro Viqueira, Jan de Vos, y Daniel Villafuerte, cuya influencia - aunque relativa en función del total de los artículos considerados — abarca prácticamente todos los campos disciplinarios localmente estudiados, destacando en cuanto a cantidad de referencias, sobre las efectuadas con respecto a autores tales como: Collier, Rus, Navarrete, Aramoni, Harvey, Leyva; Lisbona, Freyermuth, Favre, Bonfil y Ascencio. La referencia temática a estos últimos autores está presente en trabajos provenientes de la Antropología, Historia y Sociología, mas no en los procedentes de los campos disciplinarios del Desarrollo económico y la Arqueología. 
Cuadro 5. Publicación de artículos por autor (2000-2008) ${ }^{10}$

\begin{tabular}{|c|c|c|c|}
\hline Autor y dependencia & $N^{\circ}$ & Revista & Disciplina \\
\hline $\begin{array}{l}\text { Lisbona Guillén, Miguel } \\
\text { (Proimmse) }\end{array}$ & 5 & $\begin{array}{l}\text { Anuario Cesmeca (2); Liminar; } \\
\text { Pueblos y fronteras (2) }\end{array}$ & $\begin{array}{l}\text { Sociología política; } \\
\text { Antropología (3); } \\
\text { Antropología social }\end{array}$ \\
\hline $\begin{array}{l}\text { Ascencio Franco } \\
\text {,Gabriel (Proimmse) }\end{array}$ & 4 & Anuario Cesmeca (4) & $\begin{array}{l}\text { Antropología social; Historia; } \\
\text { Sociología (2) }\end{array}$ \\
\hline Reyes Gómez, Laureano & 4 & $\begin{array}{l}\text { Anuario Cesmeca; Anuario IEI } \\
\text { (3) }\end{array}$ & Antropología (4) \\
\hline $\begin{array}{l}\text { Escalona Victoria, José } \\
\text { Luis (Fac. Ciencias } \\
\text { Sociales Unach) }\end{array}$ & 4 & Anuario IEI (2); Liminar (2) & Antropología (4) \\
\hline $\begin{array}{l}\text { Paniagua Mijangos } \\
\text { Jorge (IEI-Unach) }\end{array}$ & 3 & $\begin{array}{l}\text { Anuario IEI (2); Pueblos y } \\
\text { fronteras }\end{array}$ & Antropología (3) \\
\hline $\begin{array}{l}\text { Ramos Maza Teresa } \\
\text { (CESMECA- } \\
\text { UNICACH) } \\
\end{array}$ & 3 & Liminar; Anuario Cesmeca (2) & Antropología cultural (3) \\
\hline $\begin{array}{l}\text { Aramoni Calderón } \\
\text { Dolores (IEI-Unach) }\end{array}$ & 3 & Anuario IEI; Liminar & Antropología; Historia (2) \\
\hline $\begin{array}{l}\text { Rivera Farfán Carolina } \\
\text { (Ciesas Sureste) }\end{array}$ & 3 & $\begin{array}{l}\text { Pueblos y fronteras (2); } \\
\text { Anuario Cesmeca }\end{array}$ & Antropología (2); Historia \\
\hline $\begin{array}{l}\text { Morales Barragán } \\
\text { Federico (Proimmse) }\end{array}$ & 3 & Anuario Cesmeca (2); Liminar & Desarrollo económico (3) \\
\hline $\begin{array}{l}\text { Page Pliego Jaime } \\
\text { Tomás (Proimmse) }\end{array}$ & 3 & $\begin{array}{l}\text { Anuario Cesmeca (2); Pueblos } \\
\text { y fronteras }\end{array}$ & Antropología (3) \\
\hline $\begin{array}{l}\text { Castro Apreza Inés } \\
\text { (Cesmeca-Unicach) }\end{array}$ & 3 & Anuario Cesmeca (2); Limi & $\begin{array}{l}\text { Sociología; Sociología } \\
\text { política (2) }\end{array}$ \\
\hline $\begin{array}{l}\text { Villafuerte Solís Daniel } \\
\text { (Cesmeca-Unicach) }\end{array}$ & 3 & Liminar (2); Anuario IEI & Sociología (3) \\
\hline $\begin{array}{l}\text { Rodríguez Castillo Luis } \\
\text { (Proimmse) }\end{array}$ & 3 & Anuario Cesmeca (2); Liminar & $\begin{array}{l}\text { Sociología política (2); } \\
\text { Antropología política }\end{array}$ \\
\hline $\begin{array}{l}\text { Solís Cruz Jesús } \\
\text { (Cesmeca-Unicach) }\end{array}$ & 3 & Anuario Cesmeca (2); Liminar & Antropología (3) \\
\hline $\begin{array}{l}\text { Gutiérrez Cruz Sergio } \\
\text { Nicolás }\end{array}$ & 3 & Anuario Cesmeca (2); Liminar & $\begin{array}{l}\text { Historia; Sociología; } \\
\text { Sociología de la educación }\end{array}$ \\
\hline López Jiménez Fany & 3 & $\begin{array}{l}\text { Anuario Cesmeca; Pueblos y } \\
\text { fronteras (2) }\end{array}$ & Arqueología \\
\hline $\begin{array}{l}\text { Villasana Benítez } \\
\text { Susana }\end{array}$ & 3 & Anuario IEI (3) & $\begin{array}{l}\text { Antropología (2); Informe } \\
\text { técnico }\end{array}$ \\
\hline
\end{tabular}

Fuente: Elaboración propia.

Del total de artículos (172), la autoría de poco más de 33\% de ellos recae en 17 autores. Entre estos figuran sólo algunos autores que además de ser referenciados por otros autores 
producen y publican en las revistas estudiadas, detentando posiciones importantes por situarse en los puntos de intersección de las diversas redes de comunicación conformadas tanto a través de las referencias de que son ellos objeto, como de su participación como actores que promueven de manera directa - publicando sus trabajos en las revistas consideradas - vínculos de comunicación entre ellos y con un número significativo de otros autores sobre los cuales su influencia ha quedado consignada en el Cuadro 4.

Cuadro 6. Autores que publican en las revistas estudiadas.

Número de artículos escritos en el periodo, y referencias temáticas por ellos efectuadas

\begin{tabular}{|c|c|c|}
\hline $\begin{array}{l}\text { Autor de } \\
\text { artículo }\end{array}$ & $\begin{array}{l}\text { \# Artículos } \\
\text { escritos }\end{array}$ & Cita $a$ : \\
\hline \multicolumn{3}{|l|}{$\begin{array}{l}\text { Mario } \\
\text { Humberto } \\
\text { Ruz }\end{array}$} \\
\hline $\begin{array}{l}\text { Juan Pedro } \\
\text { Viqueira }\end{array}$ & 1 & $\begin{array}{l}\text { Lisbona Miguel; Aramoni Dolores; Gage Thomas; Villa Rojas; Díaz del } \\
\text { Castillo; Calnek Edward; Códice Mendoza; Remesal; Ximénez; García } \\
\text { Vargas y Rivera; Markman; Reyes; Vázquez; Archivo General de Indias; } \\
\text { AGCA; AHDSC; Torres; Turok; Nájera; De Vos; Becerra; Ruz; Velasco; } \\
\text { MacLeod J.; Charnay; Gerhard; Villalobos }\end{array}$ \\
\hline \multicolumn{3}{|r|}{ (2) } \\
\hline $\begin{array}{l}\text { Daniel } \\
\text { Villafuerte }\end{array}$ & 3 & $\begin{array}{l}\text { Bartra; Zermeño; Meyer; Perales; Villafuerte; Piam; Prensa local y } \\
\text { nacional; Prigogine; Plan Puebla-Panamá; Salgado; Wallerstein; López; } \\
\text { Vidal; Touraine; Sen; Nigh; Inegi; Beck. } \\
\text { Villafuerte; Lisbona. } \\
\text { Calva; Villafuerte y García; Villafuerte; Inegi; Burguete; Bartra }\end{array}$ \\
\hline \multicolumn{3}{|l|}{$\begin{array}{l}\text { George } \\
\text { Collier }\end{array}$} \\
\hline Jan Rus & 2 & $\begin{array}{l}\text { Testimonios de entrevistas } \\
\text { Moermann; Prokosch }\end{array}$ \\
\hline \multicolumn{3}{|l|}{$\begin{array}{l}\text { Gonzálo } \\
\text { Aguirre }\end{array}$} \\
\hline $\begin{array}{l}\text { Dolores } \\
\text { Aramoni }\end{array}$ & 3 & $\begin{array}{l}\text { Archivo Histórico Diocesano de San Cristóbal; Thomas; Rivera; López } \\
\text { Austin; Aguirre; Ruz; Fuentes y Guzmán; Gage; Montes; Hill; Ximenez; } \\
\text { Martínez; Cervantes; Vauchez. } \\
\text { Ruz; Lenkersdorf; Ximénez; Remesal. } \\
\text { Ochiai; Lisbona; Morales; Molina; Ruz; Ximénez; Aramoni; Díaz; } \\
\text { Becerra; Viqueira; Pitarch }\end{array}$ \\
\hline $\begin{array}{l}\text { Thomas } \\
\text { Benjamin }\end{array}$ & & \\
\hline
\end{tabular}




\begin{tabular}{|l|l|l|}
\hline $\begin{array}{l}\text { Carlos } \\
\text { Navarrete }\end{array}$ & \\
\hline Neil Harvey & & \\
\hline $\begin{array}{l}\text { Xóchitl } \\
\text { Leyva }\end{array}$ & & \\
\hline $\begin{array}{l}\text { Miguel } \\
\text { Guillén }\end{array}$ & 5 & $\begin{array}{l}\text { Lisbona; Reyes; Archivo Estatal de la Comisión Agraria Mixta. } \\
\text { Prensa local; Información del Gobierno Municipal; entrevistas; } \\
\text { Expediente Justo Sierra. } \\
\text { Lisbona; Gossen; Foster; Thomas; Báez; Córdova; Reyes; Duverger; } \\
\text { Reifler; Vogt; Aguirre; Sanmartín; López; Rivera y Arrieta; Blom; Villa } \\
\text { Rojas; Reyes y Villasana; Velasco; Alonso; Pitarch. } \\
\text { Villafuerte; Villa Rojas; Viqueira; Benjamín; García; Wasserstrom; } \\
\text { Lisbona; De la Peña; Paniagua; Barragán y Link; Bataillon; Schryer; } \\
\text { Hofman; Palerm; Shadow y Rodríguez; Brading. } \\
\text { De la Cerda; Villa Rojas; Lisbona; Viqueira; Sonnleinter Willibald; } \\
\text { Vilafuerte; Inegi; referencias un periódico local }\end{array}$ \\
\hline $\begin{array}{l}\text { Graciela } \\
\text { Freyermuth }\end{array}$ & & \\
\hline Henri Favre & & \\
\hline $\begin{array}{l}\text { Guillermo } \\
\text { Bonfil }\end{array}$ & & \\
\hline $\begin{array}{l}\text { Ascencio } \\
\text { Franco }\end{array}$ & 3 & $\begin{array}{l}\text { Villafuerte; Linck; Barragán; Pulido; Tarrio; Fernández. } \\
\text { Fuentes de gobierno; estadísticas oficiales; prensa escrita y 2 entrevistas } \\
\text { a ex colaboradores del personaje. } \\
\text { Freedson y Pérez; Sánchez; De León; Muñoz; Toledo; Pérez; Viqueira; } \\
\text { Sonnleitner; Barragán; Tomé y Fábregas; Toledo; Burguete; Díaz; Rossi } \\
\text { y Krader; Alonso; Cota; Guerra; De Piña; Streeck y Smither. }\end{array}$ \\
\hline
\end{tabular}

Fuente: Elaboración propia.

El establecimiento de redes de conocimiento entre la comunidad académica estudiada se encuentra determinado más por aspectos relacionados con el grado de penetración alcanzado por aquellos autores cuyos trabajos se han sedimentado como parte de una tradición local. Son autores de origen local, nacional y mundial, cuyo impacto se refleja en el mantenimiento de enfoques y metodologías que aún no han logrado traducirse en plataformas innovadoras de conocimiento. ${ }^{11}$ La incidencia de estos autores difiere de acuerdo con su presencia en el contexto institucional.

Por un lado, figuran autores como Mario Humberto Ruz, Jan De Vos, George Collier; Gonzalo Aguirre, Thomas Benjamín, Carlos Navarrete, Neil Harvey, Xóchitl Leyva, Graciela Freyermuth, Henri Favre y Guillermo Bonfíl, cuya condición local, nacional o 
mundial, representa un factor diferencial en cuanto a su capacidad para potenciar cambios innovadores desde el punto de vista teórico y metodológico. La penetración alcanzada por sus trabajos asegura hasta cierto punto la posibilidad de introducir propuestas novedosas desde el punto de vista teórico y metodológico. Ello depende sin duda alguna de su respectiva capacidad de autocrítica, así como de la reformulación de sus respectivos parámetros de generación de saberes con la finalidad de asegurar para la comunidad académica local una posición en el debate actual sobre el futuro de las ciencias sociales.

Por otro lado, se encuentra un conjunto de autores principalmente locales que además de su incidencia en la producción académica publicada en las principales revistas de ciencias sociales de Chiapas ejercen una influencia más significativa a través de la publicación de sus propios trabajos en tales revistas. Se trata de Juan Pedro Viqueira, Daniel Villafuerte, Jan Rus, Dolores Aramoni, Miguel Lisbona y Gabriel Ascencio. Sin asegurar que sean ellos los únicos responsables de la dirección que pudiese tomar la investigación social en Chiapas, sí son ellos los garantes de la consistencia que logren alcanzar los procesos de apropiación y generación de sentido desplegados en las tareas de investigación, y con ello convertirse en los gestores del desarrollo local-global de las ciencias sociales en Chiapas. $\mathrm{Su}$ acción mediadora en los procesos de cambio institucional depende del nivel de conciencia por ellos alcanzado para determinar en qué medida su trabajo se encuentra situado o no dentro de un dominio disciplinario producente de sentido, así como el área de influencia e impacto social de los resultados obtenidos. En suma, el desafío que enfrenta este grupo de autores apunta en tres direcciones.

Primero, el conocimiento y reconocimiento de la experiencia innovadora generada por otros investigadores que, aun cuando sus trabajos - publicados en las revistas y en el periodo aquí estudiados - no acumulen un determinado número de referencias, destacan por el potencial innovador de sus resultados. Es el caso de los estudios de Araceli Burguete Cal y Mayor, María del Carmen García Aguilar, Federico Morales Barragán; Astrid Pinto Durán, Teresa Ramos Maza, Carolina Rivera Farfán, Tim Trench, Conrado Márquez Rosado, 
Miguel Ángel Hernández Molina, Shophie Hvostoff y otros entre los que figuran trabajos realizados por estudiantes de maestría. ${ }^{12}$

Segundo, promover un debate fundado en la necesidad de destradicionalizar las miradas disciplinarias, un ejercicio que obligadamente se materialice en un replanteamiento de sus respectivas prácticas de investigación y en la socialización de sus resultados. En el caso de la Historia, atender al llamado de Viqueria (2002: 42-43) y «...mostrar la infinita diversidad de respuestas a los problemas comunes y universales de la condición humana, asentar la historicidad de todo lo humano, de todo lo que conforma a las sociedades y a las culturas...». En el caso de la Sociología, evaluando de manera permanente los resultados obtenidos en la práctica de «...una Sociología del sujeto indígena más que una de la intersubjetividad, [de] una Sociología de la confrontación más que de las transacciones, [de] una Sociología de la pertenencia más que de las movilidades y [de] una Sociología de la marginación más que de la "potencia de actuar"...» (Didou 2003:116). En el ámbito del Desarrollo económico refrendar la necesidad apuntada por Valdivieso (2006: 13) y recuperar «...una mirada integral acerca de los procesos sociales, en la cual se enfatiza acerca de las interrelaciones de normas legales, sistemas de poder político, códigos de conducta, esquemas organizacionales y arreglos económicos a partir de los cuales se han estructurado, históricamente, las sociedades». Para el caso de la Antropología, considerar el llamado que desde los años noventa del siglo pasado Arizpe (1993: 13-16) sistematizó de la siguiente manera: a) La necesidad de extender los estudios de sociedad-medio ambiente a los fenómenos producidos por el cambio global: climático, pérdida de ozono, industrial, deforestación, erosión, la acidificación, etc., sin olvidar estudios sobre la disminución de diversidad cultural y la pérdida de conocimientos locales. b) En lugar del estudio de grupos indígenas y campesinos como comunidades autocontenidas, atender la relación de estos con las sociedades nacionales, los movimientos trasnacionales -migración, comunicación y el consumo, entre otros- - c) El estudio de las afectaciones enfrentadas por la microelectrónica y las telecomunicaciones, y por último, d) el reconocimiento de las nuevas maneras de conocer. 
Tercero, actualizar el sentido social de la investigación universitaria reconociendo e identificando los ángulos fundamentales de la vida social ausentes en las tareas de investigación. Algunos de estos grandes temas son: el estudio sobre la capacidad de aprendizaje — adaptación y resistencia - de los distintos grupos étnicos al margen de las visiones integracionistas formuladas desde fuera de sus respectivas comunidades; el análisis de la situación política, económica, social y cultural de Chiapas en función de los factores que a escala local, nacional e internacional intervienen de manera decisiva, modificando estructuras, formas y procesos de participación social — política, religiosa, económica, familiar, etc.—; la dinámica urbano-rural, la inversión y el desarrollo, el federalismo y la autonomía local, así como los temas mediados por necesidades sociales, tales como: salud, educación, empleo, medio ambiente, comunicación, migración y recursos naturales.

Cumplir con las condiciones aquí enunciadas arrojará, sin duda, nuevos elementos para diseñar pautas y establecer criterios que permitan optimizar el potencial de una comunidad en lo que respecta al impacto social y científico de su empresa académica. 


\section{REFERENCIAS BIBIOGRÁFICAS}

Arizpe, Lourdes, 1993, «Presentación». En Balance de la antropología en América Latina, pp. 11-17, compilado por Lourdes Arizpe y Carlos Serrano. Instituto de Investigaciones Antropológicas y Centro Regional de Investigaciones Multidisciplinarias, Universidad Nacional Autónoma de México, México.

Berger, Peter y Thomas Luckmann, 2001, La construcción social de la realidad. Amorrortu editores, Buenos Aires.

Didou, Sylvie, 2003, «Producción sobre movimiento social en las revistas de ciencias sociales en Chiapas: algunos comentarios». Liminar, v. 1, n. 1, pp. 109-119.

Gingras, Yves, 2008, «La fièvre de l'évaluation de la recherche. Du mauvais usage des faux indicateurs», pp. 1-22. CRIST, Note de recherche, Montreal.

Molina José Luis, Juan Muñoz y Philippe Losego, 2009, «Red y realidad: aproximación al análisis de las redes científicas». En http://seneca.uab.es/antropologia/jlm/ public_archivos/Oviedo.pdf [consulta: 17 de febrero de 2009].

Pacheco, M. Teresa, 2007, La investigación social en Chiapas. Complejidad e innovación. IISUE-UNAM/Proimmse-UNAM. México.

Pritchard, Alan, 1969, «Statistical bibliography or bibliometrics?». En Journal of Documentation, v.25, n.4, pp.348-349.

2008, La educación en Chiapas. Desafios para la investigación social. IISUEUNAM/Proimmse-UNAM, México. 
Science Metrix. En http://www.science-metrix.com/fr/methodes_scientometrie_t.htm [consulta: 2 de abril de 2009].

Valdivieso Canal, Susana, 2006, «¿Nuevos aires en la teoría del desarrollo?». Problemas del desarrollo, v. 37, n. 144, pp. 11-31.

Viqueira, Juan Pedro, 2002, Encrucijadas Chiapanecas. El Colegio de México/Tusquets Editores, México. 
Notas

${ }^{1}$ La realización de este trabajo fue posible gracias a apoyo institucional del Proimmse y del IISUE, ambas dependencias de la UNAM.

${ }_{2}$ «Los significados objetivados de la actividad institucional se conciben como un "conocimiento" y se transmiten como tales; una parte de este "conocimiento" se considera relevante a todos, y otra, solo a ciertos tipos. Toda transmisión requiere cierta clase de aparato social, vale decir que algunos tipos se sindican como transmisores y otros como receptores del "conocimiento" tradicional, cuyo carácter específico variará, por supuesto, de una sociedad a otra» (Berger y Luckman 2001: 93).

${ }^{3}$ Las publicaciones periódicas son: el anuario del Cesmeca y la Revista Liminar del Centro de Estudios Superiores de México y Centroamérica (Cesmeca-Unicach); el anuario del Instituto del Estudios Indígenas (IEI-Unach), la Revista Pueblos y fronteras del Programa de Investigaciones Multidisciplinarias sobre Mesoamérica y el Sureste de la Universidad Nacional Autónoma de México (Proimmse-UNAM). Cabe señalar que Pueblos y fronteras modificó su nombre partir del mes de junio de 2006, convirtiéndose desde entonces en una revista electrónica con el nombre de Pueblos y fronteras digital.

${ }^{4}$ A diferencia de los criterios internacionales fijados por el Institut of Scientific Information (Thomson ISI), las publicaciones periódicas en y sobre Chiapas con respaldo institucional no cuentan en sentido estricto con una periodicidad claramente establecida y cumplida; sus respectivos órganos editoriales están, en la mayor parte de los casos, conformados por los mismos investigadores que publican sus trabajos en dichas revistas.

${ }^{5}$ «El tratamiento y manejo de la literatura científica por medios cuantitativos de recuento y análisis sirve no solo para analizar el volumen de publicaciones, la productividad de autores, revistas o materias, sino también en un sentido más amplio, para el conocimiento de los procesos y la naturaleza de las Ciencias, para el que a partir de 1960 comienza a acuñarse el término Cienciometría o Ciencia de la Ciencia. En http://clio.rediris.es/clionet/articulos/bibliometria.htm [Consulta: 12 de septiembre de 2007].

${ }^{6}$ Ver la investigación publicada: Pacheco M. Teresa. La investigación social en Chiapas. Complejidad e innovación. IISUE-Proimmse (UNAM). México, 2007. Este trabajo ofrece un análisis sobre el impacto social y científico del contenido del material publicado sobre Chiapas en el lapso 2000-2005 en revistas locales con respaldo institucional.

${ }^{7}$ De reciente creación si se compara con la antigüedad y trayectoria de las otras tres.

${ }^{8}$ Un balance sobre el impacto social y científico del contenido de los productos de la investigación en educación en y sobre Chiapas durante el periodo 2000-2005 — publicados bajo cualquier formato de publicación institucional- se encuentra publicado en: Pacheco, M. Teresa. La educación en Chiapas. Desafíos para la investigación social. IISUE-Proimmse (UNAM). México, 2008.

${ }^{9}$ Ídem.

${ }^{10}$ No se contemplan los artículos en coautoría.

${ }^{11}$ Ver la evaluación efectuada en Pacheco, M. Teresa (2007 y 2008).

${ }^{12}$ Los trabajos realizados por estos autores pueden ser localizados en las revistas aquí consideradas y en los números publicados dentro del periodo 2000-2008.

Fecha de recepción: 6 de noviembre de 2009.

Fecha de aceptación: 19 de abril de 2010. 